\title{
EL MODELO EUROPEO EN LA MODERNIZACIÓN DE LA ESCUELA NACIONAL DE ARTES Y OFICIOS PARA HOMBRES, 1915-1932
}

\author{
Andrés Ortiz Morales ${ }^{1}$ \\ orcid.org/0000-0001-5479-3139
}

\begin{abstract}
Resumen: El propósito es atender la influencia de los modelos europeos en la construcción del sistema denominado enseñanza técnica, que derivaría en la educación superior tecnológica mexicana. Se trata de una aproximación al contacto que tuvieron algunos actores con la experiencia educativa internacional, antes y durante la reorganización de la Escuela Nacional de Artes y Oficios para Hombres (ENAOH), la cual fue incentivada por los procesos de industrialización del sistema productivo, el avance de los conocimientos científico tecnológicos y la demanda de educación por parte de la sociedad, movilizada por la Revolución Mexicana. Luego de la consulta de archivos históricos como el de la Secretaría de Educación Pública (SEP), de la Escuela Superior de Ingeniería Mecánica y Eléctrica (ESIME), de la Secretaría de Relaciones Exteriores (SRE) y de documentos producidos por la SEP y por algunos de los actores durante la época, se encontró que el interés del grupo Constitucionalista por la educación técnica, fue favorable a la ENAOH en particular, de tal manera que la administración del presidente Venustiano Carranza la transformó en 1915 en Escuela Práctica de Ingenieros Mecánicos y Electricistas (EPIME), organizando en ella las carreras de ingeniería mecánica y eléctrica, y que fue la Ciudad de México el laboratorio donde se definió el tipo de educación que constituiría la enseñanza técnica nacional. Se concluye que la reorganización de la enseñanza técnica representada por la transformación de la ENAOH en escuela profesional de ingeniería, formaba parte de un proceso de larga duración -el desarrollo de la educación superior tecnológica en el mundo industrial-, inscrito en la modernización emprendida por los Estados Nacionales en el siglo xix, tardía para el caso mexicano y en general para Latinoamérica. La experiencia internacional en materia de enseñanza técnica, fue conocida y valorada por los ideólogos de la educación en México, donde alcanzaría gran resonancia el modelo francés y la primera Ecole PolyTechnique creada en 1795, que organizó carreras fundamentadas en los conocimientos en ciencia y tecnología, ingenierías valiosas para los proyectos del Estado. Dicha experiencia cedería el paso durante la década de 1920, a la atención prestada al modelo norteamericano.
\end{abstract}

Palabras-clave: Enseñanza técnica; Educación superior tecnológica; Escuela Nacional de Artes y Oficios; Ingeniería; Modernización.

\footnotetext{
${ }^{1}$ Doctor en Historia y Etnohistoria. Presidencia del Decanato del Instituto Politécnico Nacional (México). Contacto: aortizmo@ipn.mx.
} 


\section{O MODELO EUROPEU NA MODERNIZAÇÃO DA ESCUELA NACIONAL DE ARTES Y OFICIOS PARA HOMBRES, 1915-1932}

Resumo: O objetivo é abordar a influência dos modelos europeus na construção do sistema denominado ensino técnico, que derivaria na educação superior tecnológica mexicana. Trata-se de uma aproximação ao contato que tiveram alguns atores com a experiência educativa internacional, antes e durante a reorganização da Escuela Nacional de Artes y Oficios para Hombres (Enaoh), q qual foi incentivada pelos processos de industrialização do sistema produtivo, ao avanço dos conhecimentos científicos e tecnológicos e da demanda de educação por parte da sociedade, mobilizada pela Revolução Mexicana. Pela consulta realizada em arquivos históricos, como o da Secretaría de Educación Pública (SEP), da Escuela Superior de Ingeniería Mecánica y Eléctrica (Esime), e da Secretaría de Relaciones Exteriores (SRE), e de documentos produzidos pela SEP e por outros atores à época, constata-se que o interesse do grupo Constitucionalista pelo ensino técnico foi favorável à Enaoh, de tal maneira que a administração do presidente Venustiano Carranza a transformou, em 1915, na Escuela Práctica de Ingenieros Mecánicos y Electricistas (Epime), organizando as carreiras de engenharia mecânica e elétrica, sendo a Cidade do México o laboratório onde se definiu o tipo de educação que se constituiria no ensino técnico nacional. Conclui-se que a reorganização do ensino técnico representada pela transformação da Enaoh em escola profissional de engenharia formava parte de um processo de longa duração - o desenvolvimento da educação superior tecnológica no mundo industrial -, inscrito na modernização empreendida pelos Estados Nacionais no século XIX, tardia para o caso mexicano e, em geral, para a América Latina. A experiência internacional do ensino técnico foi conhecida e valorizada pelos idealizadores da educação no México, onde alcançaria grande ressonância o modelo francês e a primeira Ecole Poly-Technique, criada em 1795, que organizou as carreiras fundamentadas nos conhecimentos em ciência e tecnologia, engenharias valiosas para os projetos do Estado. Essa experiência daria lugar, durante a década de 1920, à atenção dada ao modelo norte-americano.

Palavras-chave: Ensino técnico; Educação superior tecnológica; Escuela Nacional de Artes y Oficios; Engenharia; Modernização.

\section{Introducción}

Aquí se presenta una aproximación al contacto con la experiencia educativa internacional que tuvieron algunos actores de la reorganización de la Escuela Nacional de Artes y Oficios para Hombres (ENAOH), la cual 
fue incentivada por los procesos de industrialización del sistema productivo, el avance de los conocimientos científico tecnológicos y la demanda de educación de parte de la sociedad, movilizada por la Revolución Mexicana. Luego de la consulta de archivos históricos como el de la Secretaría de Educación Pública (SEP), de la Escuela Superior de Ingeniería Mecánica y Eléctrica (ESIME), de la Secretaría de Relaciones Exteriores (SRE) y documentos producidos por instancias de la SEP y por algunos de los actores durante la época, resulta evidente la influencia del modelo europeo en la construcción del sistema denominado enseñanza técnica, que derivaría en la educación superior tecnológica mexicana.

El modelo europeo de la educación superior tecnológica surgió sobre las cenizas de la universidad confesional cristiana, eliminada por decreto del gobierno revolucionario francés en 1793 (Gómez Oyarzú, 1998, p. 80), y se caracterizó porque recibió franco apoyo del Estado, que la vinculó a los proyectos económicos y a las necesidades materiales señaladas por él mismo; se orientó hacia la estructura ocupacional, para cubrir las necesidades de mandos medios y superiores en el sistema de producción, donde la fábrica-industria se hallaba en expansión, en detrimento del trabajo artesanal; sus contenidos unieron ciencia y técnica bajo un mismo plan de estudios, por eso la profesión de ingeniería fue representativa de esta modalidad, que llegó a adquirir el mismo rango social que las carreras liberales.

Estados Unidos asimiló en la segunda mitad del siglo xix el modelo de educación superior que hizo suya la atención de la investigación al interior de las instituciones educativas. La Johns Hopkins University, fundada en 1876, sería la primera universidad en orientarse hacia la investigación en las principales ramas científicas, motivando la renovación de las universidades más antiguas del país. Sin embargo, ni el desarrollo académico, ni los grandes logros tecnológicos ni la cercanía con México hicieron atractivo aquel país para la especialización de los ingenieros mexicanos durante la renovación de la enaoh, y explicar por qué ocurrió así es el propósito de la siguiente aproximación. 


\section{De artes y oficios a ingeniería mecánica y eléctrica}

Tras el triunfo liberal sobre el Segundo Imperio en 1867, se restableció en México el gobierno republicano de corte progresista, el cual puso en marcha las reformas que darían sustento al nuevo sistema político, económico y social. La educación, en especial de las nuevas generaciones, era fundamental para formar al ciudadano que pudiera dar continuidad al proyecto liberal, republicano y capitalista, por lo que el gobierno del presidente Benito Juárez promulgó la Ley Orgánica de Instrucción Pública en el Distrito Federal, primer paso en la organización de un sistema educativo a cargo del Ministerio de Justicia e Instrucción Pública. Al efecto, se establecieron en la Ciudad de México varias escuelas nacionales, y en el ex convento de San Lorenzo, la Escuela Nacional de Artes y Oficios para Hombres (ENAOH) abrió sus puertas en 1869, para formar artesanos y obreros que cursarían las siguientes materias: "español, francés e inglés, aritmética, álgebra, geometría, trigonometría rectilínea, física y nociones de mecánica, química general, invenciones industriales, química aplicada a las artes, economía y legislación industrial, práctica de artes y oficios en talleres que se establecieren conforme a los reglamentos que se dictaren" (Ministerio de Justicia e Instrucción Pública, 1867).

El gobierno de Porfirio Díaz (1876-1910) dio continuidad a las instituciones republicanas, pacificó al país mediante el uso de la violencia de Estado y buscó insertar la economía mexicana en el mercado mundial, mediante la exportación de materias primas, la construcción de vías de comunicación y la instalación de nuevas industrias, todo ello con el impulso de la inversión extranjera.

En su informe de 1900 el director de la ENAOH, Manuel Francisco Álvarez, reportó los talleres de carpintería, fotografía, fototipia, fundición, tornería, galvanoplastia, herrería, litografía y tipografía (Álvarez, 1900). Conforme ganaron espacios las nuevas plantas productivas mecanizadas que empleaban vapor, la fuerza hidráulica y eléctrica, la tarea educativa de la ENAOH se orientó hacia la formación de obreros especializados, aunque 
convivió con la enseñanza artesanal. Por eso, en 1907 se agregaron en el nuevo plan de estudios, los talleres de pintura decorativa industrial y escultura industrial, electricidad aplicada a la industria y mecánica aplicada a la industria (Plan de Estudios de la Escuela Nacional de Artes y Oficios para Hombres, 1907, p. 3). A pesar de esta formación, a los técnicos y artesanos mexicanos les resultaba difícil insertarse en puestos superiores en las empresas de transportes, comunicaciones, generación de electricidad, y en las explotadoras de recursos naturales básicos (minerales, petróleo, productos agrícolas), pues generalmente eran extranjeras y traían su propio personal calificado, el cual se cuidaba de no transmitir los conocimientos y habilidades que les proporcionaban status laboral. La demanda por ocupar los puestos que estaban en manos de los extranjeros se convertiría en reclamo social antes, durante y después de la Revolución Mexicana.

El proyecto de Venustiano Carranza (de 1913 a 1916 como primer jefe; de 1917 a 1920 como presidente) pretendió dar continuidad al modelo de crecimiento económico, cuidando los intereses de los particulares, de la pequeña burguesía mexicana, tanto en el campo como en las ciudades. $\mathrm{Su}$ prioridad era consolidar el papel de México como exportador de materias primas y promover la consolidación del sistema capitalista, por tanto su reforma sólo era política, de marcado nacionalismo. La transformación de la ENAOH en Escuela Práctica de Ingenieros Mecánicos y Electricistas (EPIME) en 1915-1916, puso de manifiesto el cambio de la producción artesanal hacia la industrial, se reconoció el papel de ingenieros y técnicos como encargados de aplicar los conocimientos científicos y tecnológicos en programas de modernización específicos, implementados por la iniciativa privada y protegidos por el Estado y una elite educada que admiraba el progreso alcanzado en Europa occidental y los Estados Unidos. Para modernizar las escuelas y su enseñanza, el gobierno porfirista y después el revolucionario, promovieron encuentros con los sistemas educativos extranjeros. 


\section{Europa como contrapeso a la hegemonía de Estados Unidos}

A pesar de la cercanía y del desarrollo alcanzado por el sistema educativo y la ingeniería estadounidenses, a finales del porfiriato y primeras décadas del siglo xx, los ingenieros mexicanos recurrieron a Europa para hacer estudios de especialización. El avance de los vecinos en diversos campos se admiraba pero a la vez se quería llegar a la modernidad por otros caminos, sin claudicar ante la cultura anglosajona.

A finales del siglo xix la avasalladora presencia de Estados Unidos en la vida económica de México causaba angustia entre el grupo de los científicos (intelectuales y políticos cercanos al presidente Díaz), quienes se propusieron estrechar más los vínculos con Europa; Porfirio Díaz alentaría el ingreso de capitales europeos, invertidos en deuda pública, ferrocarriles, comercio y petróleo. Esto provocó un distanciamiento con Estados Unidos en los últimos años de su gobierno; al estallar la Revolución, los norteamericanos no tomaron ninguna acción para ayudar al régimen de Díaz (Werner Tobler, 1994, p. 41).

De igual manera los contendientes en la guerra civil tuvieron que lidiar con el poderoso vecino del norte, pues su política exterior influía de manera determinante en su triunfo o derrota. Especialmente difícil fue la relación entre el gobierno estadounidense y la facción que encabezó Venustiano Carranza, pues su postura nacionalista inquietaba a los estadounidenses y a sus inversiones. La invasión al puerto de Veracruz en 1914, más el ataque de Pancho Villa a Columbus en marzo de 1916 y la expedición punitiva del ejército norteamericano que lo persiguió en México, avivaron el de por sí exaltado nacionalismo revolucionario y el sentimiento anti-yanqui de los mexicanos, que el gobierno constitucionalista fomentó mediante la prensa, sobre todo con el periódico El Demócrata. Desde el poder se propiciaba el distanciamiento entre ambos países, ocasionado por la política nacionalista mexicana, la cual nutría el imaginario colectivo y a la vez la desconfianza que la sociedad mexicana tenía hacia el norteamericano. 
La Constitución de 1917 confirmó las sospechas de los estadounidenses: el Artículo 27 Constitucional declaró como bienes de la nación el suelo, aguas y subsuelo, y facultó al Estado a cederlo en usufructo de acuerdo al bienestar nacional. Ante esta legislación, Estados Unidos aplicó un boicot comercial a México, principalmente de armas y alimentos; tampoco concedió los préstamos que le solicitó la administración de Carranza. El historiador Arnaldo Córdova afirma que el gobierno mexicano emprendió entonces una lucha diplomática contra los yanquis, y "los carrancistas y sobre todo don Venustiano, tuvieron el mérito de oponerse a las repetidas pretensiones del gobierno de EUA de someter a México incondicionalmente a sus dictados" (Córdova, 1973, p. 254). Carranza buscó de paso incrementar el control estatal sobre las empresas extranjeras (Werner Tobler, 1994, p. 199).

Más tarde, los gobiernos revolucionarios de Álvaro Obregón y Plutarco Elías Calles (1920-1928), también recurrieron a Europa en la búsqueda de una válvula que aliviara la intensa presión que ejercían los norteamericanos, quienes pretendían echar por tierra las reformas nacionalistas que se oponían a su hegemonía en Latinoamérica. La situación se hizo más tensa cuando en abril de 1926 se aprobó el reglamento del Artículo 27 Constitucional (Meyer, 1999, p. 71; 76); como resultado se instaló un estado de pre-guerra frente a Estados Unidos en el primer semestre de 1927.

La tensión se reflejó en el discurso educativo, José Vasconcelos (1920-1924), quien impulsó la creación de la Secretaría de Educación Pública de la que fue el primer titular, consideró que no debía caerse en el error de convertir la técnica en un fin, como ocurría en Estados Unidos, donde, a decir del secretario, las obras carecían de expresiones espirituales por buscar siempre la utilidad, eran ricos pero vacíos en cultura. No rechazaba el progreso, el dominio y el empleo de la tecnología, ni ignoraba los avances que esos países habían alcanzado, pero Vasconcelos proponía que México accediera a ellos sin perder en el proceso su el derecho a manifestar su identidad ${ }^{2}$.

2 Archivo Histórico de la Secretaría de Educación Pública (AH-SEP), Sección Departamento Escolar, serie DGETIC, caja 1, exp. 18, folio 2. 


\section{Encuentros con Europa en}

\section{la modernización de la ENAOH}

Durante los últimos años del régimen de Díaz, el ingeniero Félix F. Palavicini realizó un viaje por Europa y Estados Unidos entre 1906 y 1907 por encargo del ministro de Instrucción, Justo Sierra; debía observar el trabajo de las escuelas técnicas de aquellos lugares e informar y proponer reformas para modernizar ese tipo de educación en México (Monteón González, 1993, p. 121); sus recomendaciones fueron publicadas en 1909 en el libro: Las escuelas técnicas (Palavicini, 1909), donde privilegió la atención sobre las escuelas europeas. Palavicini sería responsable de la Secretaría de Instrucción Pública y Bellas Artes (SIPBA)3 del gobierno de facto de Venustiano Carranza (1913-1917); en mayo de 1915 durante el receso de las hostilidades entre constitucionalistas y la Convención, inició la reorganización de la enseñanza de la ENAOH hacia la profesionalización.

En la elaboración de los planes de estudio de la recién transformada EPIME, participó el ingeniero Manuel Luis Stampa Ortigoza, primer director del plantel y también director de Enseñanza Técnica de la SIPBA. Stampa Ortigoza estudió en Europa, en el Institut Industrielle du Nord de la France en Lille, donde se graduó de ingeniero civil y electricista en 1906 (Bazant, 1984, p. 276-278). Ese mismo año tomó cursos sobre electricidad en la Universidad de París. A fines de 1907 ya en México, en la ENAOH fue encargado de la cátedra de Electricidad Industrial y autor del libro Lecciones de electricidad industrial profesadas en la Escuela Nacional de Artes y Oficios para Hombres de México (Bazant, 1984, p. 279).

Poco después de la reforma que dio origen a la EPIME, se incorporaron a ella, casi simultáneamente, dos europeos: Rodolfo Peter Yanecic y Guillermo A. Keller Bergamini. Rodolfo Peter, de nacionalidad austriaca, estudió construcción de caminos, ferrocarriles, puentes y edificios, y construcción de fortificaciones en la Academia Superior para el Estado Mayor Técnico de Viena, y realizó estudios teóricos y prácticos para construcción

3 Creada en 1905, cuyo primer titular fue Justo Sierra. 
de máquinas en la Universidad Politécnica Imperial y Real de la misma ciudad. Llegó a México en 1911 y desempeñó varios cargos como ingeniero; en febrero de 1919 se incorporó a la Escuela Nacional de Ingenieros (ENI) como profesor de Tecnología y Resistencia de Materiales, mientras que en mayo ingresó a la epime como profesor para Proyectos de Máquinas y Turbinas de Vapor ${ }^{4}$. A poco de haberse colocado en la escuela, señaló algunas deficiencias y propuso mejoras, una de ellas la comentó así el director de la EPIME Miguel Bernard el 5 de julio de 1920:

\begin{abstract}
Nada más justo que la observación hecha por usted, respecto a que para el ingeniero mecánico-electricista tiene más interés el que conozca las reacciones y procedimientos inherentes a la producción de metales, y a la consiguiente construcción de hornos y máquinas adecuados, que la fabricación del jabón o del vinagre; por lo tanto, sírvase usted dar sus instrucciones a este respecto a los profesores. 5
\end{abstract}

Para 1920, se le expidieron tres nombramientos con los cuales atendió las clases de Proyectos de Máquinas y Turbinas, Ferrocarriles Eléctricos, y la de Máquinas Elevatorias [sic] y de Transportes. Se le comisionó además para que inspeccionara las clases de Química Elemental e Industrial, y la revisión del plan de estudios para la carrera de ingeniero mecánico. ${ }^{6}$

El suizo Guillermo A. Keller se sumó a la planta de profesores de la entonces denominada Escuela de Ingenieros Mecánicos y Electricistas (EIME) el 15 de junio de 1920. Graduado como ingeniero en la Escuela Real Industrial Alejandro Volta y en la Superior de Ingenieros de Nápoles, Italia, realizó estudios de perfeccionamiento en la Escuela de Ingenieros de Dresden y en la de Charlottenburg en el área de Berlín. Vino a México para la organización y puesta en marcha de las plantas de la Compañía Hidroeléctrica e Irrigadora de Chapala.7 En la EIME impartiría diversos cursos

\footnotetext{
4 Expediente personal de Peter Yanecic, Rodolfo, Archivo Histórico de la Escuela Superior de Ingeneiría Mecánica y Eléctrica (AH ESIME); fondo Expedientes de Personal Destacado (EPD), caja 55, fojas 17 y 19.

5 Ibidem, foja 86.

${ }^{6}$ Ibidem, fojas 358 a 365 .

7 Expediente personal de Keller Bergamini, Guillermo A., AH ESIME; fondo EPD, caja 39, fojas 19 y 20.
} 
relacionados con mecánica, magnetismo y electricidad. ${ }^{8}$ Debido a su iniciativa, en 1921 la eime contó con un Laboratorio de Electricidad y Magnetismo. 9

Ambos profesores, a decir de su alumno Manuel Cerrillo Valdivia, quien se convertiría en destacado científico- "venían con lo mejor del conocimiento de su época” (López Revilla; Reynoso, 1991, p. 285-298.).

\section{Modelo centro europeo: generar conocimientos en la educación superior}

Fruto de la Revolución Francesa, la Escuela Politécnica (1795) se dedicaría a la difusión y desarrollo de ciencia y tecnología, instrumentos del Estado-nación en la lucha por la supremacía. A pesar de la derrota de Francia, tras la restauración monárquica, naciones como Austria, Prusia, Dinamarca, Suiza y Rusia, fundaron politécnicos para renovar la enseñanza tradicional de sus universidades, como estrategia para mantenerse como potencias en el concierto de las naciones, pues sus gobiernos entendieron que ciencia y tecnología eran factores de predominio.

Durante el siglo XIX Francia se convirtió en el gran centro generador de ciencia e Inglaterra de tecnologías, seguidos por Alemania, que tras la unificación, su gobierno entendió la importancia de la ciencia para incentivar el desarrollo económico-industrial y enfrentar situaciones de guerra. De este modo los investigadores fueron contratados por el Estado en aquellas naciones que visualizaron el nexo entre ciencia y tecnología con el progreso nacional.

Además de los profesores europeos a principios de la década de 1920, la dirección de la EIME solicitó y obtuvo de la SEP pensiones para que los alumnos destacados Carlos Vallejo Márquez, Emilio Leonarz, Manuel

\footnotetext{
8 Ibidem, fojas 2 y 3.

9 Folleto histórico y conmemorativo de la ESIME, 1916-1941, México, Instituto Politécnico Nacional, Talleres Gráficos de la Dirección de Publicaciones y Materiales Educativos del IPN, reproducción facsimilar, 1997, p. 16.
} 
Sevilla Serdán, Luis Castañeda y Miguel Fonseca viajaran a estudiar a Europa, con gastos que fueron cargados al presupuesto de la Dirección General de Enseñanza Técnica, Industrial y Comercial (DGETIC) (León Olivares, 1989, p. 183). A su regreso se incorporaron como profesores de enseñanza técnica. Así lo reportó el jefe de la DGETIC en 1924 al jefe del Departamento Escolar de la SEP:

Carlos Vallejo Márquez estuvo en Zurich, Suiza especializándose en Hidrotécnica, que cursó en la Escuela Politécnica, y la práctica la hizo en varios establecimientos, entre ellos la fábrica Wyss y Cía. El señor Vallejo trajo certificados que acreditan su buen aprovechamiento; actualmente ocupa el puesto de jefe de Talleres en el Instituto Técnico Industrial. El señor Emilio Leonarz ingresó a la Montenistische Hochschul en Loeben, Austria, donde estudió Físico-Química, Metalurgia, Metalografía, Fabricación de Coke y Mineralogía. ${ }^{10}$

Carlos Vallejo trabajó como profesor en máquinas de combustión interna, y en 1929 era docente en la Facultad de Química y Farmacia de la Universidad Nacional. También ocupó el cargo de director de la Escuela Superior de Ingeniería Mecánica y Eléctrica (ESIME) en dos ocasiones (en 1932 y 1955) y fue subjefe del Departamento de Enseñanza Técnica de la SEP, donde participó en la estructuración del sistema de educación tecnológica, junto con el jefe del Departamento, Luis Enrique Erro, y del titular de la SEP, Narciso Bassols.

Guillermo Keller, Rodolfo Peter, Carlos Vallejo y Emilio Leonarz también formaron parte de la plantilla de profesores que enseñaron a los jóvenes aspirantes a ingenieros en la década de 1920; introdujeron la noción de que el ingeniero debía conocer el sustento científico que respalda toda aplicación de conocimientos e innovaciones de los países industriales en el medio mexicano. No sólo influyeron en la EPIME-EIME-ESIME, pues Keller y Vallejo trabajaron también en escuelas de la Universidad Nacional.

${ }^{10}$ AH-SEP, Sección Departamento Escolar, serie DGETIC, caja 9, exp. 12, folios 25 y 26. 
Tras el ingreso de estos personajes a la EIME se observaron algunas trasformaciones. En los planes de estudios de la carrera ingenieros mecánicos-electricistas de 1926 fue notoria la mayor carga de contenidos tanto en las materias teóricas como en las eminentemente prácticas; se puede encontrar una mayor atención a las matemáticas, la física y la química. Mientras que en 1916 se impartían Academia de Física y Física Industrial en toda la carrera, en 1926 se tenían Física elemental, Física 1er curso, Física $2^{\circ}$ Curso y Laboratorio de Física; en 1916 se contaba con dos cursos anuales de química, en tanto que en 1926 además de éstos se agregó un Laboratorio de química en el $4^{\circ}$ año. También la introducción de los laboratorios es indicador de la importancia que se le asignó a la práctica como experiencia de aprendizaje, reflejo del incremento de los espacios curriculares relacionados con la práctica.

\section{Consideraciones finales}

Se concluye que la reorganización de la enseñanza técnica representada por la transformación de la ENAOH en escuela profesional de ingeniería, formaba parte de un proceso de larga duración -el desarrollo de la educación superior tecnológica en el mundo industrial-, inscrito en la modernización emprendida por los Estados nacionales en el siglo XIX, tardía para el caso mexicano y en general para Latinoamérica.

Se encontró que el interés del grupo Constitucionalista por la educación técnica, propició la transformación de la ENAOH, tras 48 años de existencia, en Escuela Práctica de Ingenieros Mecánicos y Electricistas (EPIME). La administración de Venustiano Carranza, hábil constructor de legislación para disminuir al enemigo, renovó esta institución en 1915, en plena contienda revolucionaria, organizando en ella las carreras de ingeniería mecánica y eléctrica, también como medida propagandística para mostrar su compromiso con la modernización nacional.

A pesar de que los revolucionarios criticaron acremente la tendencia de los beneficiados por el sistema porfirista por los lujos y la moda fran- 
cesa, la solidez del sistema educativo de Francia y de sus institutos de investigación, propiciaron que los viajes de estudio a ese y otros países europeos, continuaron aún después de la caída de Porfirio Díaz, financiados por las primeras administraciones revolucionarias. La experiencia internacional en materia de enseñanza técnica, fue conocida y valorada por los ideólogos de la educación en México, donde alcanzaría gran resonancia el modelo francés y la primera Ecole Poly-Technique creada en 1795, que organizó carreras fundamentadas en los conocimientos en ciencia y tecnología, ingenierías valiosas para los proyectos del Estado.

Sin embargo esta orientación habría de cambiar cuando Europa occidental perdió su centralidad, al resentir los estragos de la guerra y quedar arruinada su economía, además de la persecución que en la década de 1930 emprenderían los nazis en contra de la comunidad científica relacionada con los judíos, provocando el desmantelamiento del grupo de científicos que mantenían la delantera en Física en Europa central. En 1932 la eime adquirió el nombre de Escuela Superior de Ingeniería Mecánica y Eléctrica esime, y en esa década, también como reflejo de una nueva relación entre México y su vecino del norte, la formación en el extranjero de los mexicanos se enfocaría definitivamente hacia los Estados Unidos, y en el caso la ingeniería, de manera destacada hacia el Instituto Tecnológico de Massachusetts. Queda por abordar este interesante proceso.

\section{Referencias}

\section{Archivos}

Archivo Histórico de la Escuela Superior de Ingeniería Mecánica y Eléctrica ah ESIME, fondos: Escuela Superior de Ingeniería Mecánica y Eléctrica (ESIME); Serie Expedientes de Personal Destacado (EPD); Tomás Guzmán Cantú (TGC).

Archivo Histórico Central del Instituto Politécnico Nacional (AHC-IPN), fondo: Ex directores Generales (EG); Histórico de Personal (HP). 
Archivo Histórico de la Secretaría de Educación Pública (AH-SEP), sección: Departamento Escolar, serie Dirección General de Enseñanza Técnica, Industrial y Comercial (DGETIC).

Archivo Histórico de la Secretaría de Relaciones Exteriores (SRE).

\section{Bibliografía y documentos}

Álvarez, M. Informe de labores, 31 de diciembre de 19oo, en Archivo Histórico de la Escuela Superior de Ingeniería Mecánica y Eléctrica (AHESIME).

Bazant, M. "La enseñanza y la práctica de la ingeniería durante el Porfiriato" en Historia Mexicana, revista trimestral publicada por el Centro de Estudios Históricos de El Colegio de México, v. XXXIII, n. 3, enero-marzo de 1984, p. 276-278.

Carrera Stampa, M. et al., Libro de oro conmemorativo de la Escuela Superior de Ingeniería Mecánica y Eléctrica. México: Litográfica Universo, 1967.

Córdova, A. La ideología de la Revolución Mexicana: la formación del nuevo régimen. México: Era, 1973.

Escuelas del Departamento de Enseñanza Técnica, Industrial y Comercial (Estampas), México: Secretaría de Educación Pública, Departamento de Enseñanza Técnica, Industrial y Comercial, 1924.

Folleto histórico y conmemorativo de la ESIME, 1916-1941, México: Instituto Politécnico Nacional, Talleres Gráficos de la Dirección de Publicaciones y Materiales Educativos del IPN, reproducción facsimilar, 1997.

Gómez Oyarzú, G. La universidad a través del tiempo. México: Universidad Iberoamericana, 1998.

Lazarín Miranda, F. La política para el desarrollo. Las escuelas técnicas industriales y comerciales en la Ciudad de México, 1920-1932. México: Universidad Autónoma Metropolitana, 1996.

León Olivares, B. La Escuela de Ingenieros Mecánicos y Electricistas, un análisis histórico. México, tesis de maestría, Instituto Politécnico Nacional, PESTYC, 1989.

López Revilla, R.; Reynoso, R. "La génesis del Cinvestav: Entrevista con Eugenio Méndez Docurro”, Avance y Perspectivas, v. 10, octubre-diciembre de 1991

Meneses Morales, E. Tendencias educativas oficiales en México, 19111934. México: Centro de Estudios Educativos, Universidad Ibero Americana, 1998. 
Meyer, L. México y el mundo. Historia de sus relaciones exteriores. México: Senado de la República, tomo VI, 1999.

Ministerio de Justicia e Instrucción Pública, Ley Orgánica de Instrucción Pública en el Distrito Federal, 2 diciembre de 1867.

Monteón González, H. (coord.). La esime en la historia de la enseñanza técnica. Primer tramo. México: Instituto Politécnico Nacional, 1993.

Moreno Botello, R. La escuela del proletariado. La educación técnica industrial en México. 1876-1938. México, Universidad Autónoma de Puebla, 1987.

Padua, J. Educación, industrialización y progreso técnico en México. México: El Colegio de México, UNESCO, 1984.

Palavicini, F. F. Las escuelas técnicas. México: Talleres de imprenta y ramos anexos, Fiat Lux, 1909.

Piqueras Arenas, J. A. El taller y la escuela en la Valencia del siglo XIX. Valencia: Ayuntamiento de Valencia, 1983.

Plan de Estudios de la Escuela Nacional de Artes y Oficios para Hombres, México, Tipografía Económica, 1907.

Rodríguez Álvarez, M. Á.; Krongold, M. 50 años en la historia de la educación tecnológica. México, Instituto Politécnico Nacional, 1988.

Ruiz Larraguivel, E. Ingenieros en la industria manufacturera. México: Universidad Nacional Autónoma de México, Centro de Estudios sobre la Universidad, Plaza y Valdés, 2004.

Sánchez Meneses, M. J. Escuela Nacional de Artes y Oficios para Hombres: discurso y vida cotidiana (1867-1915), tesis de maestría, Instituto Politécnico Nacional, Coordinación General de Posgrado e Investigación, Centro de Investigaciones Económicas, Administrativas y Sociales, 2003. Secretaría de Estado y del Despacho de Justicia e Instrucción Pública, "Programas y textos que deben regir el año escolar de 1903 y la Escuela Nacional de Artes y Oficios para Hombres", en Boletín de Instrucción Pública, Órgano de la Secretaría del Ramo, Secretaría de Estado y del Despacho de Justicia e Instrucción Pública, México, 1903.

SIPBA. Programas para la Escuela Nacional de Artes y Oficios de Mujeres en 1906 y textos para la clase de Elementos de Historia Natural en la misma escuela, en Boletín de Instrucción Pública, Secretaría de Instrucción Pública y Bellas Artes, México, 1906.

. Boletín de Instrucción Pública, órgano de la Secretaría del Ramo, tomo xiv, México, Tipografía Económica, 1910.

. Boletín de Educación, órgano de la Secretaría de Instrucción

Pública y Bellas Artes; tomo 1, México, noviembre de 1915, n. 2. 
SEP. Boletín de la Secretaría de Educación Pública, tomo 1, n. 2, México, SEP, 6 de marzo de 1922.

. Boletín de la Secretaría de Educación Pública, 1er semestre de 1923; tomo I; n. 3 y 4, México, SEP, 1923.

. Boletín de la Secretaría de Educación Pública, tomo IV; n. 3, 4, 6 y 7, México, SEP, 1925.

. Boletín de la Secretaría de Educación Pública, tomo vi; n. 9 y 10, México, SEP, 1927.

. El esfuerzo educativo en México. La obra educativa del go-

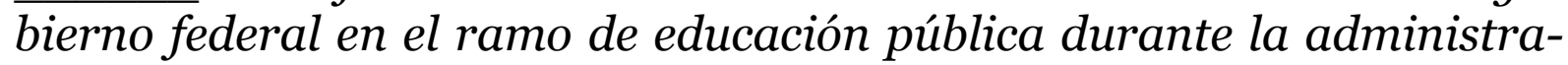
ción del Presidente Plutarco Elías Calles (1924-1928); tomo I, México, Publicaciones de la Secretaría de Educación Pública, 1928.

. Memoria que indica el estado que guarda el ramo de educación Pública, el 31 de agosto de 1930; presentada por el Lic. Aarón Sáenz, secretario del ramo, para conocimiento del H. Congreso de la Unión, en obediencia del Artículo 93 Constitucional; México, SEP, 1930.

. Memoria relativa al estado que guarda el ramo de educación pública el 31 de agosto de 1932, tomo I, México, Talleres Gráficos de la Nación, SEP, 1932.

Werner Tobler, H. La revolución Mexicana: transformación social y cambio político, 1876-1940. México: Alianza, 1994. 